\title{
Anti-glomerular basement membrane glomerulonephritis and thrombotic microangiopathy in first degree relatives; a case report
}

\author{
Thomas Idorn ${ }^{1 *}$, Lone Schejbel ${ }^{2}$, Casper Rydahl ${ }^{3}$, James Goya Heaf ${ }^{3}$, Karen Riis Jølvig², Marie Bergstrøm²,
} Peter Garred ${ }^{2}$ and Anne-Lise Kamper ${ }^{1}$

\begin{abstract}
Background: Anti-glomerular basement membrane glomerulonephritis and thrombotic microangiopathy are rare diseases with no known coherence.

Case Presentation: A daughter and her biological mother were diagnosed with pregnancy-induced thrombotic microangiopathy and anti-glomerular basement membrane glomerulonephritis, respectively. Both developed end-stage renal disease. Exploration of a common aetiology included analyses of HLA genotypes, functional and genetic aspects of the complement system, ADAMTS13 activity and screening for autoantibodies.

The daughter was heterozygous carrier of the complement factor I G261D mutation, previously described in patients with membranoproliferative glomerulonephritis and atypical haemolytic uremic syndrome. The mother was non-carrier of this mutation. They shared the disease associated complement factor H silent polymorphism Q672Q (79602A > G).

Conclusion: An unequivocal functional or molecular association between these two family cases was not found suggesting that the patients probably share another, so far undiagnosed and unknown, predisposing factor. It seems highly unlikely that two infrequent immunologic diseases would occur by unrelated pathophysiological mechanisms within first degree relatives.
\end{abstract}

Keywords: Aetiology, Anti-glomerular basement membrane glomerulonephritis, Atypical haemolytic-uremic syndrome, Thrombotic microangiopathy

\section{Background}

Goodpasture's disease or anti-glomerular basement membrane glomerulonephritis (anti-GBMGN) has an incidence around one/million/year [1]. Autoantibodies are directed against epitope(s) in the glomerular basement membrane, in response to unknown stimuli [2]. It occurs in siblings and twins and a strong association with the major histocompatibility complex class II gene HLA DR2, different HLA DRB1 genes, HLA DR15 and DR4

\footnotetext{
* Correspondence: thomas.idorn@rh.regionh.dk

'Department of Nephrology, Rigshospitalet, University of Copenhagen, Blegdamsvej 9, DK-2100 Copenhagen $\varnothing$, Denmark

Full list of author information is available at the end of the article
}

has been reported, while DR1 and DR7 seem to protect $[3,4]$.

Thrombotic microangiopathy (TMA) is characterized by haemolytic anaemia, thrombocytopenia and organ injury due to platelet thrombosis in the microcirculation. Depending on predominantly kidney or CNS location, it is classified as haemolytic-uremic syndrome (HUS) or thrombotic thrombocytopenic purpura (TTP). HUS may be typical HUS or atypical HUS (aHUS) including the pregnancy induced form. The incidence of acquired TMA is $17.5 / \mathrm{mil}-$ lion/year, 17\% occur during pregnancy or postpartum [5]. aHUS has defects in complement regulation causing increased alternative pathway activation in glomerular vessels. The causes are disabling mutations in the genes of 
complement factor $\mathrm{H}(\mathrm{CFH})$, membrane cofactor protein (MCP; CD46), complement factor I (CFI) and thrombomodulin $(T H B D)$, and enabling mutations in factor $\mathrm{B}$ and $\mathrm{C} 3$ [6]. Autoantibodies against $\mathrm{CFH}$ sometimes occur [7]. Dense deposit disease (formerly membranoproliferative glomerulonephritis type II) and C3 glomerulonephritis are also associated with uncontrolled complement activation and mutations in $C F H$ or CFI have been described [8]. The same mutations have occasionally been found in both $\mathrm{C} 3$ glomerulonephritis and aHUS [9]. This, in combination with incomplete penetrance of aHUS, indicates that other genetic or environmental factors trigger these diseases.

Anti-GBMGN has been associated with TMA (in particular TTP) and immune complex glomerulonephritis. Both diseases were diagnosed in the same person, typically with TMA diagnosed shortly after anti-GBMGN debut $[10,11]$. To our knowledge anti-GBMGN has never been associated with aHUS and anti-GBMGN and TMA has never been described within families. We present a daughter and her biological mother diagnosed with pregnancy-induced TMA and anti-GBMGN respectively, with 14 years in between and describes our exploration of a suspected link between these two rare diseases.

\section{Case presentation}

\section{Daughter}

A 22-year-old female was admitted with acute kidney failure following abruptio placentae causing severe vaginal haemorrhage in gestation week 33, resulting in stillbirth. The patient had a history of abortions in gestation weeks 9 and 28. Placental infarction at the second miscarriage resulted in heparin treatment during the actual pregnancy, which had been normotensive without proteinuria 2 days before admission.

She presented with haemolytic anaemia, thrombocytopenia, schistocytes, uraemia, hypertension, oliguria and proteinuria (Table 1). Treatment included plasmapheresis, haemodialysis and glucocorticoids. Anti-cardiolipin immunoglobulins, anti-Scl-70 and anti-double stranded DNA were negative. Anti-GBM and ANCA titers were not examined at disease onset, but were negative when examined 14 years later. Renal biopsy showed necrotic glomeruli with thrombi, platelet deposits and fibrin, vascular changes with luminal narrowing and intimal thickening, but no deposits. The diagnosis was TMA (pathologist Thomas Horn, MD, DMSc, Herlev Hospital, Denmark) (Figure 1). A grand mal seizure occurred during hospitalization (no simultaneous metabolic derangements or severe hypertension). The clinical diagnosis was pregnancy-induced TMA with components of aHUS and TTP. After 6 months, kidney function improved and the patient was temporarily dialysis-independent for three years. She was never transplanted.

\section{Mother}

A 69-year-old female was admitted with anuria and acute kidney failure. The patient had a 6-year hypertension history, hypothyroidism for 30 years and a minor stroke 4 months earlier treated by carotid thrombendarterectomy. At that time p-creatinine was normal. Blood tests showed severe uraemia and anaemia, but no haemolysis (Table 1). The anti-GBM titer was positive, $95 \mathrm{U} / \mathrm{mL}$ (ELISA-kit, Wieslab, Sweden; ELISA-reader TECAN, Switzerland) and myeloperoxidase anti-neutrophil cytoplasmic antibodies titer (MPO ANCA) was $25 \mathrm{U} / \mathrm{mL}$. Proteinase-3 ANCA was negative (ELISA-reader TECAN, Switzerland) and chest X-ray normal. Treatment included methylprednisolone, cyclophosphamide, plasmapheresis and haemodialysis. Renal biopsy showed diffuse extracapillary glomerulonephritis with predominantly fresh crescentic formations, focal and segmental necrosis and linear deposition of IgG along the glomerular basement membrane, consistent with anti-GBMGN (pathologist Claus B. Andersen MD, DMSc, Rigshospitalet, Denmark) (Figure 2). The anti-GBM and MPO ANCA titres normalised after 16 days. There were no signs of TMA at any time. Kidney function was not regained.

\section{Additional analyses}

Following maternal diagnosis, additional information was obtained and the following analyses were done:

Family history: Further elaboration of the family history of kidney disease was done in order to identify additional cases. No other family members had ever experienced clinically significant kidney diseases or any signs of kidney disease. It was not possible to obtain blood samples for genetic analyses from the father. Mother and daughter lived in the same household until a few years before disease onset of the daughter. There were no obvious exposures to environmental factors such as drugs, hydrocarbons or other toxins. They discharged different occupations.

HLA tissue typing (Dynabeads, Dynal, Norway): HLA$A 1,26 ; B 7,8 ; D R 2,3$ (mother), HLA-A1;B8,37;Cw6;DR3,6 (daughter). Subtyping was not performed. ADAMTS13 activity and antibodies (CytoFlour ${ }^{\circledR} 4000$ Fluorescence Plate Reader, Applied Biosystems Inc, USA): ADAMTS13-protein 0.61 U/L (0.75-1.33) (mother); $0.97 \mathrm{U} / \mathrm{L}$ (daughter). No antibodies. The complement system (Wielisa, Wieslab, Sweden): Both patients had normal alternative- and classical pathway function. The daughter had reduced Mannose-binding lectin (MBL) pathway activity, and additional gene typing (SSP-PCR) revealed gene type $\mathrm{XA} / \mathrm{B}$, compatible with a severely reduced $\mathrm{MBL}$ level. Gene sequencing of $C F H, C F I, M C P$ and THBD showed heterozygosity for a CFI mutation (G261D, 
Table 1 Baseline data

\begin{tabular}{|c|c|c|c|}
\hline Compound and Unit - SI units & Reference & Daughter, (TMA/aHUS) & Mother, (anti-GBMGN) \\
\hline Haemoglobin, mmol/L (g/L) & $7.0-10.0(113-161)$ & $5.3(85)$ & $6.1(98)$ \\
\hline Platelets, $\times 10^{9} / \mathrm{L}$ & $150-400$ & 46 & 533 \\
\hline Haptoglobin, $\mu \mathrm{mol} / \mathrm{L}$ & $4-23$ & $<5$ & 13 \\
\hline Lactate dehydrogenase, U/L & $150-450$ & 7724 & 313 \\
\hline Bilirubin, $\mu \mathrm{mol} / \mathrm{L}$ & $4-17$ & 36 & 9 \\
\hline Peripheral smear & & $>5 \%$ Schistocytes (Coombs test not performed) & $\mathrm{n} / \mathrm{a}$ \\
\hline White blood cell count $\times 10^{9} / \mathrm{L}$ & $3.0-9.0$ & 18.3 & 21.0 \\
\hline C-reactive protein, $\mathrm{nmol} / \mathrm{L}$ & $<95$ & $\mathrm{n} / \mathrm{a}$ & 981 \\
\hline Activated partial thromboplastin time, seconds & $23-35$ & 49 & 26 \\
\hline Factor II + VII + X, U/L & $>0.60$ & 1.00 & 0.85 \\
\hline Creatinine, $\mu \mathrm{mol} / \mathrm{L}$ & $40-110$ & 430 & 799 \\
\hline Urea nitrogen, $\mathrm{mmol} / \mathrm{L}$ & $2.5-7.5$ & 15.0 & 30.7 \\
\hline Potassium, mmol/L & $3.5-5.0$ & 6.6 & 5.9 \\
\hline Sodium, mmol/L & $136-146$ & 137 & 131 \\
\hline Phosphorus (inorganic), mmol/L & $0,80-1,50$ & 1,99 & 2,37 \\
\hline Calcium, mmol/L & $2,20-2,60$ & 2,18 & 2,15 \\
\hline Albumin, $g / L$ & $36-48$ & 28 & 37 \\
\hline Bicarbonate, mmol/L & $23-31$ & 20 & 21 \\
\hline
\end{tabular}

Baseline data at admission. Reference interval according to Danish standards.

alternative syntax G243D) and several disease risk associated polymorphisms in the daughter. The mother was non-carrier of the G261D mutation but shared the disease associated CFH Q672Q silent polymorphism (Table 2). CFH-autoantibodies were not investigated, but none of the patients were carriers of the common CFHR1/CFHR3 deletion or other CFHR1 deletions strongly associated with antibody induced aHUS [12]. Measurement of C3 levels were not performed.

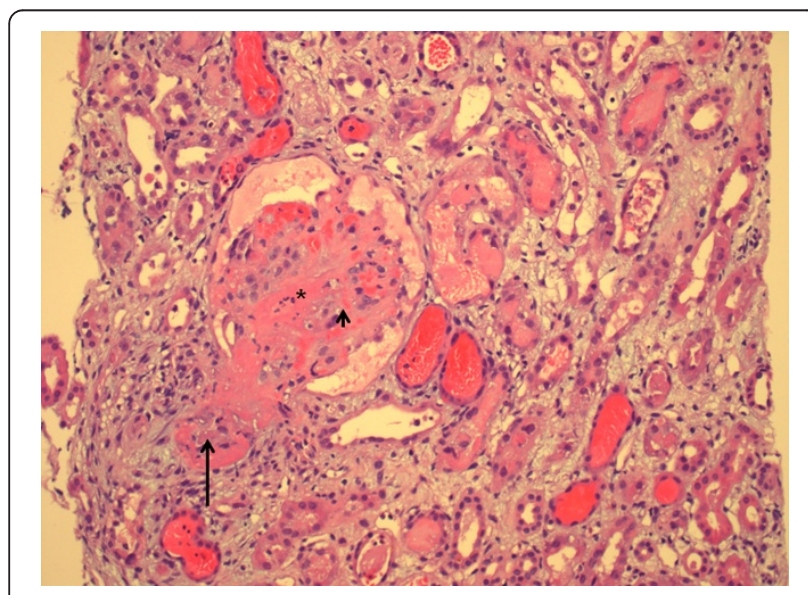

Figure 1 TMA/aHUS. Light microscopy, x200, hematoxylin and eosin stain. Thrombosis of the afferent arteriole ( $\uparrow$, large arrow) and partial necrosis of the glomerulus with deposition of fibrin $\left(^{*}\right)$ and fragmented erythrocytes ( $\uparrow$, small arrow).

\section{Conclusions}

The occurrence of two very rare renal diseases within the same family is likely to be explained by a common aetiology. One linkage could be a common autoantibodyprofile; however this was not demonstrated. ANCApositive testing in anti-GBMGN occurs in 30\% [13] and HLA tissue typing demonstrated known predisposing relations without any conspicuous shared patterns. The mother's slightly reduced ADAMTS13-protein is probably

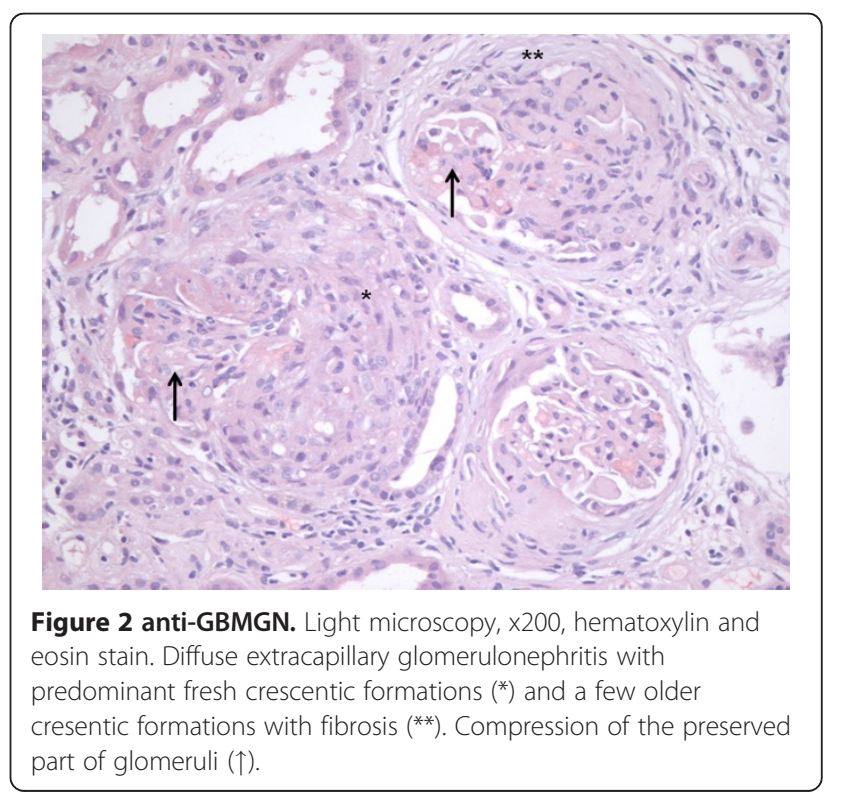


Table 2 Genetic analyses

\begin{tabular}{|c|c|c|c|c|c|}
\hline Gene & Variation & Description & Disease association & Daughter, (TMA/aHUS) & Mother (anti-GBMGN) \\
\hline \multirow[t]{9}{*}{ CFI } & NG_007569 & & & & \\
\hline & c. $5256 \mathrm{~A}>\mathrm{G}$ & Intron rs7671905 & Polymorphism & homozygous & homozygous \\
\hline & c. $42455 C>T$ & Intron rs79375065 & Polymorphism & heterozygous & Non-carrier \\
\hline & c.46524G >A & Intron rs4382037 & Polymorphism & homozygous & homozygous \\
\hline & c. $46615 G>A$ & G261D & Mutation reported in MPGN and aHUS & heterozygous & Non-carrier \\
\hline & c.49140C >A & Intron rs 7437142 & Polymorphism & homozygous & homozygous \\
\hline & c.49159ins AATTT & Intron rs78629056 & Polymorphism & homozygous & homozygous \\
\hline & c. $57335 C>G$ & Intron rs7441380 & Polymorphism & homozygous & homozygous \\
\hline & c.66205C>T & Intron rs551 & Polymorphism & homozygous & homozygous \\
\hline \multirow[t]{6}{*}{$\mathrm{CFH}$} & NG_007259.1 & & & & \\
\hline & C.38184A>C & A307A & Disease risk polymophism (AMD) & heterozygous & Non-carrier \\
\hline & c.43097C>T & $\mathrm{H} 402 \mathrm{Y}$ & Disease risk polymophism (AMD) & heterozygous & Non-carrier \\
\hline & c.79602A>G & Q672Q & Disease risk polymorphism (aHUS) & heterozygous & heterozygous \\
\hline & c.89786C $>$ A & Intron rs375046 & Polymorphism & heterozygous & heterozygous \\
\hline & $c .93634 G>T$ & E936D & Disease risk polymorphism (aHUS) & heterozygous & Non-carrier \\
\hline \multirow[t]{4}{*}{ MCP } & NG_009296 & & & & \\
\hline & c. $12610 A>G$ & L139L rs12126088 & Rare non-Disease Causing Polymorphism & heterozygous & Non-carrier \\
\hline & c. $20790 G>T$ & Intron rs 2724374 & Polymorphism & heterozygous & Non-carrier \\
\hline & c.36158G >A & Intron rs1962149 & Polymorphism & heterozygous & Non-carrier \\
\hline \multirow[t]{2}{*}{$T H B D$} & NG_012027 & & & & \\
\hline & c. $6578 \mathrm{C}>\mathrm{T}$ & A473V rs1042579 & Polymorphism & homozygous & heterozygous \\
\hline
\end{tabular}

Genetic screening for variation in the $\mathrm{CFI}$-, $\mathrm{CFH}$-, MCP- (CD46) and THBD-genes. Position of the variations in the respective GeneBank refrence sequences as well as dbSNP numbers are listed.

$C F I=$ Complement Factor I, CFH = Complement Factor $\mathrm{H}, M C P=$ Membrane Cofactor Protein, $T H B D=$ Thrombomodulin, $A M D=$ Age-related Macula Degeneration.

not significant. However, the daughter's normal ADAMTS13 activity and antibody absence during screening, does not exclude affection at diagnosis. The daughter's G261D CFI-gene mutation combined with diseaseassociated polymorphisms may predispose to the pregnancy-induced TMA. The G261D mutation has been found in other patients with $\mathrm{C} 3$ glomerulonephritis and aHUS $[9,14]$. Despite several tests by Nilsson et al., no functional effect of the G261D mutation on CFI mediated complement regulation or on CFI serum levels could be demonstrated [14]; however, it may have effects not revealed by the in vitro tests, or be a marker for a linked genetic deficiency. The silent G672G polymorphism in both patients is strongly associated with aHUS [15], and even though this is unlikely to be the shared aetiology, it is possible that it influenced the disease in both patients. Reduced MBL activity was demonstrated in the daughter but not the mother. This has no known relation to TMA (or anti-GBMGN).

In conclusion, we have found a shared $C F H$ polymorphism that may confer increased complement-mediated disease risk, but no other connection between TMA and anti-GBMGN. The diseases may require multiple triggers including mutations, polymorphisms, autoantibodies and perhaps infections, and a common genetic susceptibility cannot be ruled out.

\section{Consent}

Written informed consent was obtained from both patients for publication of this case report and accompanying images of the kidney biopsies. Acceptance was noted in the patients' records. A copy of the written consent is available for review by the Editor-in Chief of BMC Nephrology.

\section{Abbreviations}

anti-GBMGN: Anti-glomerular basement membrane glomerulonephritis; TMA: Thrombotic microangiopathy; TTP: Thrombotic thrombocytopenic purpura; aHUS: Atypical HUS; CFH: Complement factor H; CFI: Complement factor I; MCP: Membrane cofactor protein; THBD: Thrombomodulin.

\section{Competing interests}

The authors have no financial or non-financial competing interests and no disclosures.

\section{Authors' contributions}

TI: Participated in research design, collection of data and blood samples, information of the patients, data analysis and writing of the paper. LS: Participated in research design, gene analyses, data analysis and writing of the paper. CR: Participated in collection of data and blood samples, information of the patients and writing of the paper. JGH: Participated in research design, information of the patients, data analysis and writing of the paper. KRJ: Participated in gene analyses and writing of the paper. MB: Participated in gene analyses and writing of the paper. PG: Participated in research design, gene 
analyses, data analysis and writing of the paper. ALK: Participated in research design, data analysis and writing of the paper. All authors have read and approved the final version of the article.

\section{Acknowledgements}

None. Financial support was not applied for.

\section{Author details}

'Department of Nephrology, Rigshospitalet, University of Copenhagen, Blegdamsvej 9, DK-2100 Copenhagen Ø, Denmark. ${ }^{2}$ Department of Clinical Immunology, Rigshospitalet, University of Copenhagen, Blegdamsvej 9, DK-2100 Copenhagen Ø, Denmark. ${ }^{3}$ Department of Nephrology, Herlev Hospital, University of Copenhagen, Herlev Ringvej 75, DK-2730 Herlev, Denmark.

Received: 23 January 2012 Accepted: 26 July 2012

Published: 26 July 2012

\section{References}

1. Bolton WK: Goodpasture's syndrome. Kidney Int 1996, 50:1753-1766.

2. Pusey CD: Anti-glomerular basement membrane disease. Kidney Int 2003, 64:1535-1550.

3. Phelps RG, Rees AJ: The HLA complex in Goodpasture's disease: a model for analyzing susceptibility to autoimmunity. Kidney Int 1999, 56:1638-1653.

4. Rees AJ, Peters DK, Compston DA, Batchelor JR: Strong association between HLA-DRW2 and antibody-mediated Goodpasture's syndrome. Lancet 1978, 1:966-968.

5. Terrell DR, Williams LA, Vesely SK, Lammle B, Hovinga JA, George JN: The incidence of thrombotic thrombocytopenic purpura-hemolytic uremic syndrome: all patients, idiopathic patients, and patients with severe ADAMTS-13 deficiency. J Thromb Haemost 2005, 3:1432-1436.

6. Kavanagh D, Goodship T: Genetics and complement in atypical HUS. Pediatr Nephrol 2010, 25:2431-2442.

7. Moore I, Strain L, Pappworth I, Kavanagh D, Barlow PN, Herbert AP, et al: Association of factor $\mathrm{H}$ autoantibodies with deletions of CFHR1, CFHR3, CFHR4, and with mutations in CFH, CFI, CD46, and C3 in patients with atypical hemolytic uremic syndrome. Blood 2010, 115:379-387.

8. Zipfel PF, Smith RJ, Skerka C: Factor I and factor H deficiency in renal diseases: similar defects in the fluid phase have a different outcome at the surface of the glomerular basement membrane. Nephrol Dial Transplant 2009, 24:385-387.

9. Servais A, Fremeaux-Bacchi V, Lequintrec M, Salomon R, Blouin J, Knebelmann B, et al: Primary glomerulonephritis with isolated $C 3$ deposits: a new entity which shares common genetic risk factors with haemolytic uraemic syndrome. J Med Genet 2007, 44:193-199.

10. Borza DB, Netzer KO, Leinonen A, Todd P, Cervera J, Saus J, et al: The goodpasture autoantigen. Identification of multiple cryptic epitopes on the NC1 domain of the alpha3(IV) collagen chain. J Biol Chem 2000, 275:6030-6037.

11. Torok N, Niazi M, Al AY, Taleb M, Taji J, Assaly R: Thrombotic thrombocytopenic purpura associated with anti-glomerular basement membrane disease. Nephrol Dial Transplant 2010, 25:3446-3449.

12. Zipfel PF, Mache C, Muller D, Licht C, Wigger M, Skerka C: DEAP-HUS: deficiency of CFHR plasma proteins and autoantibody-positive form of hemolytic uremic syndrome. Pediatr Nephrol 2010, 25:2009-2019.

13. Jayne DR, Marshall PD, Jones SJ, Lockwood CM: Autoantibodies to GBM and neutrophil cytoplasm in rapidly progressive glomerulonephritis. Kidney Int 1990, 37:965-970.

14. Nilsson SC, Karpman D, Vaziri-Sani F, Kristoffersson AC, Salomon R, Provot F, et al: A mutation in factor I that is associated with atypical hemolytic uremic syndrome does not affect the function of factor I in complement regulation. Mol Immunol 2007, 44:1835-1844.

15. Caprioli J, Castelletti F, Bucchioni S, Bettinaglio P, Bresin E, Pianetti G, et al: Complement factor $\mathrm{H}$ mutations and gene polymorphisms in haemolytic uraemic syndrome: the C-257T, the A2089G and the G2881T polymorphisms are strongly associated with the disease. Hum Mol Genet 2003, 12:3385-3395.

\section{Submit your next manuscript to BioMed Central and take full advantage of:}

- Convenient online submission

- Thorough peer review

- No space constraints or color figure charges

- Immediate publication on acceptance

- Inclusion in PubMed, CAS, Scopus and Google Scholar

- Research which is freely available for redistribution 